\title{
Predictive dosimetric parameters for gastrointestinal toxicity with hypofractioned radiotherapy in pancreatic adenocarcinoma
}

This article was published in the following Dove Press journal:

OncoTargets and Therapy

26 April 2016

Number of times this article has been viewed

Xian Liu*

Gang Ren*

Liqin $\mathrm{Li}$

Tingyi Xia

Department of Radiation Oncology, Air Force General Hospital, Beijing, People's Republic of China

*These authors contributed equally to this work
Correspondence: Tingyi Xia

Department of Radiation Oncology, Air Force General Hospital, No 30 Fucheng Road, Haidian District, Beijing I00I42, People's Republic of China

Fax +86 I0 68434886

Email xiatingyi1959@sina.com
Abstract: To better guide the development and optimization of radiotherapy planning, to reduce the incidence of radiation reactions, and to improve the quality of life of the patients with pancreatic cancer using radiotherapy, we conducted this study to explore the dosimetric parameters that predict the risk of gastrointestinal (GI) toxicity with hypofractioned radiotherapy for pancreatic cancer. Between January 2014 and January 2015, the medical records of 68 patients with pancreatic cancer who underwent helical tomotherapy at the Air Force General Hospital were analyzed. The doses delivered to the planning target volume, clinical target volume, and gross tumor volume-internal gross tumor volume of the primary pancreatic lesions were 50, 60, and 70-80 Gy in 15-20 fractions, respectively. GI toxicity was scored according to version 4.0 of the National Cancer Institute Common Terminology Criteria for Adverse Events. The stomach and duodenum were contoured separately to determine the dose-volume histogram parameters. Univariate and multivariate analyses were adopted to identify clinical and physical risk factors associated with GI toxicity. The median follow-up was 9 months (range: 4-16 months). Eighteen patients had grade II acute GI toxicity, one patient had grade III acute GI toxicity, 17 patients had grade II late GI toxicity, and one patient had grade III late GI toxicity. On univariate analysis, the volume, the average dose $D_{\text {mean }}$, the maximum dose to $1,3,5$, and $10 \mathrm{~cm}^{3}$ of the stomach and duodenum $(D 1, D 3, D 5$, and $D 10)$, and the relative volumes receiving 5-40 Gy (V5-V40), and the absolute volumes receiving 5-45 Gy (aV5-aV45) of the duodenum were significantly associated with grade II or higher GI toxicity $(P<0.05)$. On multivariate analysis, a $V 45$ of the duodenum was an independent predictor for grade II or higher GI toxicity $(P=0.031)$. The receiver operating characteristic analysis also showed that an aV45 of $0.5 \mathrm{~cm}^{3}$ was the optimal threshold to predict GI toxicity for the entire cohort. Our findings indicate that many dosimetric parameters of the duodenum correlate with grade II or higher GI toxicity. To reduce GI toxicity, the absolute volume of the irradiated duodenum should be reduced.

Keywords: pancreatic cancer, gastrointestinal toxicity, helical tomotherapy, hypofractioned radiation

\section{Introduction}

Pancreatic cancer (PC) is an extremely malignant tumor with poor prognosis. Radiotherapy (RT) has become an effective treatment modality for both early and locally advanced PC. ${ }^{1-4}$ However, the intimate connection between the pancreas and the gastrointestinal (GI) tract places the normal organs, especially the stomach and duodenum, surrounding the pancreas at significant risk for radiation GI toxicity. Thus, GI toxicity is the most important dose-limiting factor and a possible disadvantage of 
intensive RT. ${ }^{5,6}$ How to improve the efficacy of RT for PC without an increase in GI toxicity remains a clinical problem in need of resolution.

The progress made in radiation technology has enabled the use of tomotherapy (TOMO) in tumor radiation. Xia et $\mathrm{al}^{7}$ have combined the advantage of a high conformity index, high-dose rate, good focus, and good normal tissue protection of TOMO with 10 years of rich clinical experience in treatment with a gamma knife in patients with PC to propose a TOMO hypofractioned RT model for pancreatic adenocarcinoma with doses of 50, 60, and 70-80 Gy, respectively, delivered to the planning target volume (PTV), clinical target volume (CTV), and gross tumor volume (GTV)-internal gross tumor volume (IGTV) of the primary pancreatic lesions. The hypofractioned RT model proposed changes the conventional dose segmentation model with 2 Gy fractions and has demonstrated that the hypofractioned radiation model improves the tumor local dose with acceptable radiation-induced GI toxicity. To better guide the development and optimization of RT planning, to reduce the incidence of radiation GI toxicity, and to improve quality of life, we conducted this study to explore the dosimetric parameters that predict the risk of radiation-induced GI toxicity with hypofractioned RT for PC.

\section{Patients and methods}

\section{Patient characteristics}

Patients with PC were enrolled in this study between January 2014 and January 2015 with TOMO hypofractioned RT at the Department of Radiation Oncology, Air Force General Hospital, People's Republic of China. The main eligibility criteria for the analyzed patients were defined as follows: 1) age: 20-90 years; 2) Karnofsky performance status (KPS) score: $\geq 70 ; 3$ ) patients who did not undergo surgical resection of the duodenum or stomach; 4) patients with accessible dosevolume histogram (DVH) dosimetric parameters; 5) patients who have received no previous RT in the abdomen; and 6 ) patients who have completed radiation planning. After the exclusion of 34 patients, 68 patients were included. Of the 68 patients, 19 had pathologic diagnoses, and the other 49 patients were diagnosed based on symptoms, signs, and imaging studies. Tumor-node-metastasis (TNM) staging was based on version 7 of the American Joint Committee on Cancer (AJCC) Cancer Staging Manual. Of the 68 patients, two were in stage I, eight in stage II, 32 in stage III, and the remaining 26 were in stage IV. The patient and treatment characteristics are listed in Table 1. Verbal informed consent was obtained from all patients. The study was approved by the institutional review board and ethics committee of the Air Force General Hospital.
Table I Patient and treatment characteristics

\begin{tabular}{|c|c|}
\hline Characteristic & Values \\
\hline \multicolumn{2}{|l|}{ Age, years } \\
\hline Median (range) & $59.5(26-85)$ \\
\hline \multicolumn{2}{|l|}{ Sex, n (\%) } \\
\hline Female & $31(46)$ \\
\hline Male & $37(54)$ \\
\hline \multicolumn{2}{|l|}{ Location, n (\%) } \\
\hline Head/neck & $45(66)$ \\
\hline Body/tail & $23(34)$ \\
\hline \multicolumn{2}{|l|}{ T stage, n (\%) } \\
\hline TI & $2(3)$ \\
\hline $\mathrm{T} 2$ & $8(12)$ \\
\hline T3 & $14(2 \mid)$ \\
\hline $\mathrm{T} 4$ & $44(64)$ \\
\hline \multicolumn{2}{|l|}{$\mathrm{N}$ stage, $\mathrm{n}(\%)$} \\
\hline No & $38(56)$ \\
\hline NI & $30(44)$ \\
\hline \multicolumn{2}{|l|}{ RT dose, Gy } \\
\hline GTV dose, median (range) & $70(60-80)$ \\
\hline Dose per fraction, median (range) & $3.75(2.8-5.33)$ \\
\hline \multicolumn{2}{|l|}{ Chemotherapy, n (\%) } \\
\hline Concurrent & $29(43)$ \\
\hline None & $39(57)$ \\
\hline \multicolumn{2}{|l|}{ Acute toxicity, n (\%) } \\
\hline Grade II & $18(26)$ \\
\hline Grade III & $I(I)$ \\
\hline \multicolumn{2}{|l|}{ Late toxicity, n (\%) } \\
\hline Grade II & $17(25)$ \\
\hline Grade III & $\mathrm{I}(\mathrm{I})$ \\
\hline
\end{tabular}

Abbreviations: GTV, gross tumor volume; RT, radiotherapy.

\section{Radiation therapy}

Patients were administered a 3\% contrast orally before the computed tomography (CT) scan and the scan was performed 15 minutes thereafter. All of the patients underwent simulated radiation treatment in the supine position with their arms up, immobilized using a thermoplastic body cast. Contrast CT scans were performed. A set of transverse images (Figure 1) was collected at $4 \mathrm{~mm}$ thick intervals and the data were transferred to a treatment-planning station.

For treatment planning, dosimetric constraints for normal tissues were specified for the liver, kidneys, stomach, duodenum, bowel, and spinal cord. The GTV included the primary tumor and surrounding metastatic lymph nodes. The IGTV was defined as the GTV minus $3 \mathrm{~mm}$ when the maximum diameter of the GTV was $>5 \mathrm{~cm}$. The CTV was defined as the GTV plus a $5 \mathrm{~mm}$ isotropic margin. The internal target volume (ITV) was determined by adding a $5 \mathrm{~mm}$ horizontal margin and a $10 \mathrm{~mm}$ craniocaudal margin to the CTV. A further margin of $3 \mathrm{~mm}$ was added to create the PTV.

The doses delivered to the PTV, CTV, and GTV-IGTV of the primary pancreatic lesions were 50, 60, and 70-80 Gy in 15-20 fractions, respectively. The stomach was constrained by the following: maximum point dose $D_{\max }<60 \mathrm{~Gy}$; 

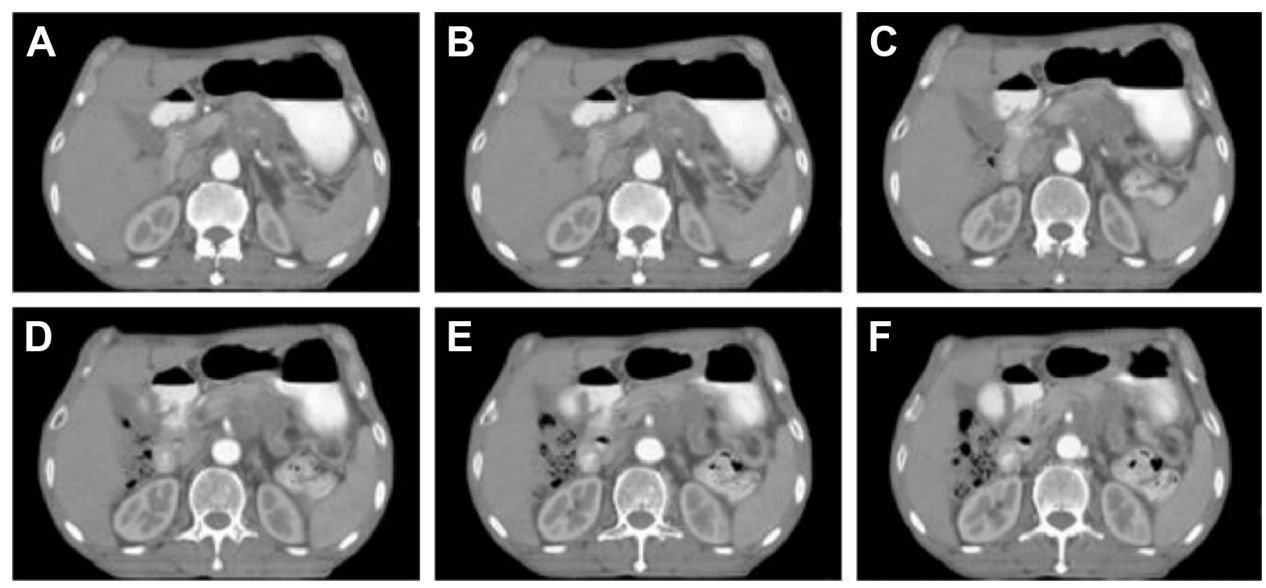

Figure I Transverse images from head to foot for a representative patient with pancreatic adenocarcinoma. Note: (A-F) Continuous CT scan images with a thickness of $4 \mathrm{~mm}$ from head to foot. Abbreviation: $\mathrm{CT}$, computed tomography.

$D 1 \leq 55 \mathrm{~Gy} ; D 3 \leq 50 \mathrm{~Gy} ; D 5 \leq 45 \mathrm{~Gy} ;$ and $D 10 \leq 40 \mathrm{~Gy}$. The duodenum was constrained by the following: $D_{\max }<55 \mathrm{~Gy}$; $D 1 \leq 50 \mathrm{~Gy} ; D 3 \leq 45 \mathrm{~Gy} ; D 5 \leq 40 \mathrm{~Gy}$; and $D 10 \leq 35 \mathrm{~Gy}$. The liver was constrained by the following: $V 30<40 \%$; and the average dose $D_{\text {mean }} \leq 20$ Gy. The $D_{\max }$ of the spinal cord was $<45$ Gy and the $V 20$ of the kidneys was $<50 \%$.

\section{Dosimetric and clinical parameters}

The following dosimetric parameters were analyzed: the GTV; dose per fraction and biological effective dose (BED); the maximum point dose $D_{\text {max }}$; the average dose $D_{\text {mean }}$; the maximum dose to $1,3,5$, and $10 \mathrm{~cm}^{3}$ of the stomach and duodenum ( $D 1, D 3, D 5$, and $D 10)$; and the absolute volume (a $V$ dose) and relative volume ( $V$ dose) of the stomach and duodenum receiving $5,10,15,20,25,30,35,40,45$, and $50 \mathrm{~Gy}$. Details regarding patient age, sex, concurrent chemotherapy, adjuvant chemotherapy, CA199 level before RT, tumor location, and the volume of tumor were also analyzed.

\section{Toxicity evaluation}

GI toxicity was scored according to version 4.0 of the National Cancer Institute Common Terminology Criteria for Adverse Events (CTCAE). ${ }^{8}$ Acute toxicity was defined as toxicity that occurred within 3 months from the beginning of RT, and late toxicity was defined as toxicity that occurred 3 months after RT. All GI toxicity instances were identified from the starting date of RT.

\section{Statistical analysis}

All data were computed using SPSS (Version 20.0 for Windows; IBM Corporation, Armonk, NY). A $P$-value $<0.05$ was accepted as significant. A chi-square test was used to analyze the count data. Spearman correlation analysis was used to analyze the relationship between occurrence of GI toxicity and the clinical and physical factors. Logistic regression models were used to identify risk factors associated with GI toxicity. The performances of the different DVH parameters in the prediction of GI toxicity were assessed by constructing the receiver operating characteristic (ROC) curve. The predictive value of a parameter was evaluated using the area under the ROC curve (AUC), and the parameter with the highest AUC represented the optimal predictor.

\section{Results}

\section{Characteristic of patients}

The median follow-up was 9 months (range: 4-16 months). The median time to the occurrence of late grade II GI toxicity was 3 months (range: 3-9 months). Sixty-one (89\%) patients had acute GI toxicity, whereas 37 (54\%) patients developed late GI toxicity. Nineteen (27\%) patients had grade II or higher acute GI toxicity. Of these 19 patients, 18 (26\%) had grade II acute GI toxicity and one (1\%) had grade III acute GI toxicity. Eighteen (26\%) patients had grade II or higher late GI toxicity. Of these 18 patients, 17 (25\%) had grade II late GI toxicity and one (1\%) had grade III late GI toxicity. No patient had grade IV or higher GI toxicity. The patient characteristics are shown in Table 1.

\section{Spearman correlation analysis for factors associated with Gl toxicity}

On univariate analysis, the volume, $D_{\text {mean }}, D 1, D 3, D 5, D 10$, the $V$ dose receiving 5-40 Gy $(V 5-V 40)$, and the a $V$ doses receiving 5-45 Gy (a $V 5-\mathrm{a} V 45)$ of the duodenum were significantly associated with grade II or higher GI toxicity $(P<0.05)$. Other factors, such as age, sex, clinical stage, concurrent chemotherapy, adjuvant chemotherapy, CA199 
Table 2 Spearman correlation analysis for factors associated with grade II or higher GI toxicity

\begin{tabular}{ll}
\hline Clinical variable & $P$-value \\
\hline Age, years & $0.75 \mathrm{I}$ \\
Sex & 0.586 \\
Clinical stage & 0.901 \\
Concurrent chemotherapy & 0.064 \\
Location & 0.207 \\
CAI99 level before radiotherapy & 0.834 \\
Volume of the tumor & 0.334 \\
Dosimetric variable & \\
BED & 0.842 \\
Prescribed dose & 0.893 \\
Dose per fraction & 0.789 \\
Duodenal DVH & \\
$D_{\text {mean }}$ & 0.002 \\
$D_{\text {max }}$ & 0.063 \\
$D I, D 3, D 5, D I 0$ & $\mathrm{~S}$ \\
V5-V40 & $\mathrm{S}$ \\
aV5-aV45 & $\mathrm{S}$ \\
V45-V50 & $\mathrm{NS}$ \\
aV50 & 0.140 \\
Gastric DVH & \\
$D_{\text {mean }}$ & 0.742 \\
$D_{\text {max }}$ & 0.213 \\
DI, D3, D5, DI0 & $\mathrm{NS}$ \\
aV5-aV50 & $\mathrm{NS}$ \\
\hline Notes DI, D3, D5, DI0, maxmy & $\mathrm{NS}$ \\
\hline
\end{tabular}

Notes: DI, D3, D5, DI0, maximum dose to I, 3, 5, and $10 \mathrm{~cm}^{3}$ of the stomach and duodenum; $D_{\max }$, maximum point dose; $D_{\text {mean }}$, the average dose.

Abbreviations: $V$, relative volume; $a$, absolute volume; $B E D$, biological effective dose; CA199, carbohydrate antigen 19-9; DVH, dose-volume histogram; GI, gastrointestinal; NS, not significant; S, significant.

level before RT, BED, the volume and location of the tumor, the prescribed dose and dose per fraction, gastric DVH, $V 45-V 50$, and a V50 of the duodenum, had no correlation with grade II or higher GI toxicity based on univariate analysis $(P>0.05$, Table 2$)$.

\section{Multiple logistic regression analysis for factors associated with GI toxicity}

Multivariate logistic regression analysis was performed with the factors that had a $P$-value $<0.01$ in the Spearman correlation analysis. Only the aV45 of the duodenum was an independent predictor for grade II or higher GI toxicity $(P<0.05$, Table 3$)$. ROC analysis was performed with

Table 3 Multiple logistic regression analysis for factors associated with grade II or higher GI toxicity

\begin{tabular}{lllllll}
\hline $\begin{array}{l}\text { Variable of } \\
\text { duodenum }\end{array}$ & B & SE & Wals & $P$-value & HR & $95 \% \mathbf{C l}$ \\
\hline aV45 & 1.959 & 0.909 & 4.643 & 0.031 & 7.091 & $1.194-42.122$ \\
\hline
\end{tabular}

Abbreviations: B, regression coefficient; aV45, absolute volume receiving $45 \mathrm{~Gy}$; $\mathrm{Cl}$, confidence interval; $\mathrm{Gl}$, gastrointestinal; $\mathrm{HR}$, hazard ratio; $\mathrm{SE}$, standard error.

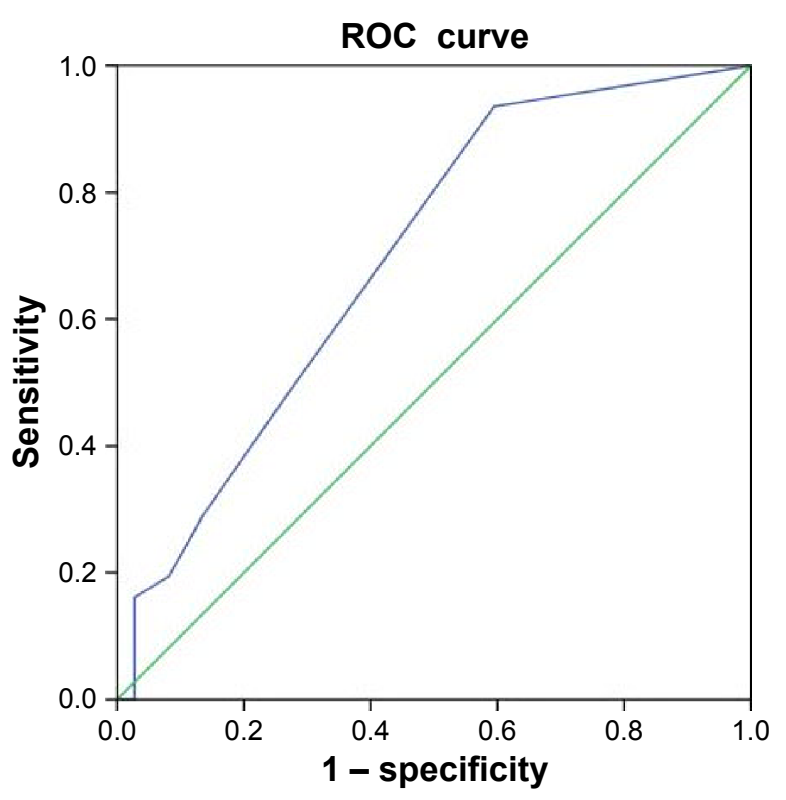

Figure 2 ROC curve of duodenal aV45 in diagnosis of grade II or higher GI toxicity.

Abbreviations: aV45, absolute volume receiving $45 \mathrm{~Gy}$; GI, gastrointestinal; ROC, receiver operating characteristic.

significant dosimetric factors, which showed that the a $V 45$ of the duodenum was an independent predictor for grade II or higher GI toxicity (AUC $=0.697, P=0.005$; Figure 2 ). The ROC analysis also showed that an a $V 45$ of $0.5 \mathrm{~cm}^{3}$ was the optimal threshold to predict grade II or higher GI toxicity for the entire cohort. The grade II or higher GI toxicity rate was $11.76 \%(2 / 17)$ versus $56.86 \%(29 / 51)$ for an a $V 45 \leq 0.5 \mathrm{~cm}^{3}$ and an a $V 45>0.5 \mathrm{~cm}^{3}$, respectively $\left(\chi^{2}=10.454, P=0.001\right)$. There was no correlation between grade III or higher GI toxicity and dosimetric factors $(P>0.05)$.

\section{Discussion}

This study confirmed a dose-dependent relationship between the irradiated duodenum and the development of grade II or higher GI toxicity after RT for pancreatic adenocarcinoma with hypofractioned radiation. Our results showed that $\geq$ grade II acute GI toxicity occurred in $27 \%$ of the patients and that $\geq$ grade II late GI toxicity occurred in $26 \%$ of the patients. The a $V 45$ of the duodenum was the best predictive factor for grade II or higher GI toxicity after RT for pancreatic adenocarcinoma with hypofractioned radiation.

In general, rates of radiation-induced GI toxicity after PC have not been consistently reported in the literature. Cattaneo et $\mathrm{al}^{9}$ conducted a trial involving 61 patients following combined chemotherapy and moderately hypofractionated rotational RT for locally advanced pancreatic adenocarcinoma. The radiation dose of 38 patients was 44.25 Gy in 
15 fractions. The other 23 patients received a simultaneous integrated boost with doses ranging from 48-58 Gy. Cattaneo et $\mathrm{al}^{9}$ reported that $33 \%$ of the patients $(n=20)$ had at least one grade II-III GI toxicity. Among these 20 patients, three had grade III toxicity. The higher occurrence of toxicity in the study by Cattaneo et $\mathrm{al}^{9}$ than in our study may be attributed in part to concurrent chemotherapy. Most of the patients were subjected to concomitant chemoradiation with oral capecitabine $\left(1,250 \mathrm{mg} / \mathrm{m}^{2} / \mathrm{d} ; \mathrm{n}=55\right.$ [89\%]) or 5-fluorouracil (250 mg/m²/d; $\mathrm{n}=5$ [8\%]).

Many studies ${ }^{9-14}$ have demonstrated that the dosimetric factor is a good indicator to predict the occurrence of GI toxicity; however, due to differences in RT fractionation, chemotherapy segment, and the patient population studied, dosimetric predictors are inconsistently reported in the literature. Currently, the following predictive dosimetric parameters have been reported: $D_{\max }, V 5, V 10, V 15, V 20$, $V 25, V 35$, and $V 50 .^{9-14}$

The most extensive analysis of GI toxicity following RT was reported by Huang et al, ${ }^{10}$ who retrospectively analyzed data from 46 patients with locally advanced PC who were treated with definitive chemoradiation and concurrent full-dose gemcitabine. For the patients treated with RT and gemcitabine alone, the radiation dose was reached at 36-42 Gy in 2.4-2.8 Gy fractions. For those patients treated with RT with concurrent gemcitabine and erlotinib, the radiation dose was given at 30-38 Gy in 2 Gy fractions. Huang et $\mathrm{al}^{10}$ reported that the $V 25$ of the duodenum was an independent predictor for GI toxicity. Nevertheless, 37\% $(n=17)$ patients experienced grade III or higher GI toxicity, which was nearly 12 times the $3 \%$ occurrence rate in our research. These higher rates of GI toxicity may be attributed to the use of high-dosage gemcitabine $\left(1,000 \mathrm{mg} / \mathrm{m}^{2}\right.$ weekly). Indeed, several studies ${ }^{15,16}$ have reported that high-dose gemcitabine increases GI toxicity compared with low-dose gemcitabine.

The significant relationship between GI toxicity and DVH parameters reported herein is in agreement with the results of two previous papers. Kelly et $\mathrm{al}^{13}$ conducted a study involving 106 patients with unresectable PC. Seventy-eight patients were treated with 50.4 Gy in 28 fractions. Twenty-eight patients received dose-escalated RT (range: 57.5-75.4 Gy in $28-29$ fractions). This study demonstrated that a $V 55>1 \mathrm{~cm}^{3}$ was the best predictor of grade II or higher duodenal toxicity. Another study by Cattaneo et $\mathrm{al}^{9}$ was a retrospective TOMO trial in which 61 patients underwent combined chemotherapy and moderately hypofractionated rotational RT for locally advanced pancreatic adenocarcinoma. The radiation dose of
61 patients was $44.25 \mathrm{~Gy}$ in 15 fractions. The $V 40$ and $V 45$ of the duodenum were strongly correlated with grade II or higher GI toxicity. The optimal V40 and V45 cutoff values were $16 \%$ and $2.6 \%$, respectively. Despite the important differences in the fractionation schemas, chemotherapeutic agents, and the patient population studied, these two studies support our results of a dose-volume relationship between the irradiated duodenum and clinically relevant GI toxicity.

Apart from the dosimetric parameters that were verified to play a key role in the prediction of GI toxicity, we also analyzed a number of clinical factors. The findings showed that for factors, such as age, sex, clinical stage, concurrent chemotherapy, adjuvant chemotherapy, carbohydrate antigen 19-9 (CA199) level before RT, BED, and the volume and location of the tumor, there was no correlation with grade II or higher GI toxicity based on univariate analysis, as previous studies reported..$^{13,14,17}$ In contrast, De Lange et $\mathrm{al}^{18}$ reported that concurrent gemcitabine may increase the GI toxicity rate; however, there was no correlation between concurrent chemotherapy and GI toxicity in our study, which may be a reflection of the low rate and low dose of concurrent chemotherapy.

There were several limitations to this study. First, this was a retrospective study conducted at one institution. Any conclusion revealed here needs to be demonstrated prospectively. Second, the condition of the duodenum and stomach are inconsistent in every treatment. Additionally, the GI toxicity was scored according to subjective symptoms, and the GI toxicity rates might be higher if all patients underwent posttreatment endoscopy. Despite this limitation, a strong correlation between the physical dose and duodenal toxicity was demonstrated.

\section{Conclusion}

In conclusion, this study confirmed a dose-dependent relationship between the duodenum and the development of grade II or higher GI toxicity after hypofractionated RT for pancreatic adenocarcinoma. Our findings indicate that many dosimetric parameters of the duodenum correlate with grade II or higher GI toxicity. To reduce GI toxicity, the a $V$ of the duodenum irradiated should be reduced. The predictive factors could be used in the optimization of RT treatment planning and in helping patients to improve their quality of life.

\section{Acknowledgment}

This work was supported by the Capital Health Development Special Scientific Research Project (2014-1-5124). 


\section{Disclosure}

The authors report no conflicts of interest in this work.

\section{References}

1. Wang J, Xia TY, Wang YJ, Guo Y. Long-term results of gamma raybased stereotactic body radiation therapy in treatment of medically unfit or inoperable non-metastatic pancreatic adenocarcinoma. Int $J$ Radiat Oncol Biol Phys. 2012;84(S3):815-816.

2. Xia T, Li HQ, Wang J, et al. Clinical outcome of hypofractionated radiation therapy for lung, liver, and pancreatic cancer. Int $J$ Radiat Oncol Biol Phys. 2010;78(S3):574-575

3. Ikeda M, Ioka T, Ito $\mathrm{Y}$, et al. A multicenter phase II trial of $\mathrm{S} 1$ with concurrent radiation therapy for locally advanced pancreatic cancer. Int J Radiat Oncol Biol Phys. 2013;85(1):163-169.

4. Ren G, Xia T, Wang Y. IMRT with capecitabine in advanced pancreatic cancer. In regard to Passoni et al. Int J Radiat Oncol Biol Phys. 2014; 89(2):431.

5. McGinn CJ, Zalupski MM, Shureiqi I, et al. Phase I trial of radiation dose escalation with concurrent weekly full-dose gemcitabine in patients with advanced pancreatic cancer. J Clin Oncol. 2001;19(22):4202-4208.

6. Small W Jr, Berlin J, Freedman GM, et al. Full-dose gemcitabine with concurrent radiation therapy in patients with nonmetastatic pancreatic cancer: a multicenter phase II trial. J Clin Oncol. 2008;26(6): 942-947.

7. Xia T, Chang D, Wang Y, Li J. Dose escalation to target volumes of helical tomotherapy for pancreatic cancer in the phase I-II clinical trial. Int J Radiat Oncol Biol Phys. 2013;87(2):S303.

8. National Cancer Institute. Common Terminology Criteria for Adverse Events (CTCAE), Version 4.0. Rockville: National Cancer Institute; 2009.

9. Cattaneo GM, Passoni P, Longobardi B, et al. Dosimetric and clinical predictors of toxicity following combined chemotherapy and moderately hypofractionated rotational radiotherapy of locally advanced pancreatic adenocarcinoma. Radiother Oncol. 2013;108(1):66-71.
10. Huang J, Robertson JM, Ye H, Margolis J, Nadeau L, Yan D. Dosevolume analysis of predictors for gastrointestinal toxicity after concurrent full-dose gemcitabine and radiotherapy for locally advanced pancreatic adenocarcinoma. Int J Radiat Oncol Biol Phys. 2012;83(4): $1120-1125$.

11. Nakamura A, Shibuya K, Matsuo Y, et al. Analysis of dosimetric parameters associated with acute gastrointestinal toxicity and upper gastrointestinal bleeding in locally advanced pancreatic cancer patients treated with gemcitabine-based concurrent chemoradiotherapy. Int $J$ Radiat Oncol Biol Phys. 2012;84(2):369-375.

12. Murphy JD, Christman-Skieller C, Kim J, Dieterich S, Chang DT, Koong AC. A dosimetric model of duodenal toxicity after stereotactic body radiotherapy. Int J Radiat Oncol Biol Phys. 2010;78(5): $1420-1426$.

13. Kelly P, Das P, Pinnix CC, et al. Duodenal toxicity after fractioned chemoradiation for unresectable pancreatic cancer. Int J Radiat Oncol Biol Phys. 2013;85(3):143-149.

14. Yoon H, Oh D, Park HC, et al. Predictive factors for gastroduodenal toxicity based on endoscopy following radiotherapy in patients with hepatocellular carcinoma. Strahlenther Onkol. 2013;189(7):541-546.

15. Crane $\mathrm{CH}$, Abbruzzese JL, Evans DB, et al. Is the therapeutic index better with gemcitabine-based chemoradiation than with 5-fluorouracilbased chemoradiation in locally advanced pancreatic cancer? Int $J$ Radiat Oncol Biol Phys. 2002;52:1293-1302.

16. Loehrer PJ Sr, Feng Y, Cardenes H, et al. Gemcitabine alone versus gemcitabine plus radiotherapy in patients with locally advanced pancreatic cancer: an Eastern Cooperative Oncology Group trial. J Clin Oncol. 2011;29:4105-4112.

17. Simpson DR, Song WY, Moiseenko V, et al. Normal tissue complication probability analysis of acute gastrointestinal toxicity in cervical cancer patients undergoing intensity modulated radiation therapy and concurrent cisplatin. Int J Radiat Oncol Biol Phys. 2012;83(1):81-86.

18. De Lange SM, van Groeningen CJ, Meijer OW, et al. Gemcitabineradiotherapy in patients with locally advanced pancreatic cancer. Eur J Cancer. 2002;38(9):1212-1217.
OncoTargets and Therapy

\section{Publish your work in this journal}

OncoTargets and Therapy is an international, peer-reviewed, open access journal focusing on the pathological basis of all cancers, potential targets for therapy and treatment protocols employed to improve the management of cancer patients. The journal also focuses on the impact of management programs and new therapeutic agents and protocols on

\section{Dovepress}

patient perspectives such as quality of life, adherence and satisfaction The manuscript management system is completely online and includes a very quick and fair peer-review system, which is all easy to use. Visit http://www.dovepress.com/testimonials.php to read real quotes from published authors. 